\title{
Optical design of the STAR-X telescope
}

\author{
Timo T. Saha ${ }^{\mathrm{a}}$, William W. Zhang ${ }^{\mathrm{a}}$, and Ryan S. McClelland ${ }^{\mathrm{b}}$ \\ ${ }^{a}$ NASA/Goddard Space Flight Center, 8800 Greenbelt Road, Greenbelt, MD 20771 \\ ${ }^{\mathrm{b}}$ SGT, Inc, 7701 Greenbelt Road Suite 400, Greenbelt MD 20770
}

\begin{abstract}
Top-level science goals of the Survey and Time-domain Astrophysical Research eXplorer (STAR-X) include: investigations of most violent explosions in the universe, study of growth of black holes across cosmic time and mass scale, and measure how structure formation heats majority of baryons in the universe. To meet these goals, the field-ofview of the telescope should be about 1 square-degree, the angular resolution should be 5 arc-seconds or below across large part of the field-of-view. The on-axis effective area at $1 \mathrm{KeV}$ should be about $2,000 \mathrm{~cm}^{2}$. Payload cost and launch considerations limit the outer diameter, focal length, and mass to 1.3 meters, 5 meters, and 250 kilograms, respectively. Telescope design is based on a segmented meta-shell approach we have developed at Goddard Space Flight Center for the STAR-X telescope. The telescope shells are divided into 30-degree segments. Individual telescopes and meta-shells are nested inside each other to meet the effective area requirements in $0.5-6.0 \mathrm{KeV}$ range. We consider WolterSchwarzschild, and Modified-Wolter-Schwarzschild telescope designs as basic building blocks of the nested STAR-X telescope. These designs offer an excellent resolution over a large field of views. Nested telescopes are vulnerable to stray light problems. We have designed a multi-component baffle system to eliminate direct and single-reflection light paths inside the telescopes. Large number of internal and external baffle vane structures are required to prevent stray rays from reaching the focal plane. We have developed a simple ray-trace based tool to determine the dimensions and locations of the baffles. In this paper, we present the results of our trade studies, baffle design studies, and optical performance analyses of the STAR-X telescope.
\end{abstract}

Keywords: Optical design, x-ray optics, x-ray mirrors, wide-field x-ray telescopes

\section{INTRODUCTION}

Field-of-view requirements, optical performance requirements, and energy range of the STAR-X telescope make the telescope design challenging [1,2]. Grazing incidence configuration limits the aperture area to very narrow annuli. To meet effective area requirements, several telescopes must be nested inside each other. Nested telescopes need to be designed so that adjacent mirrors do not block each other and obstruct the field-of-view. In addition, off-axis PSFs of individual telescopes emanating from the same object point must fall at the same field point in the image space.

$\mathrm{X}$-ray telescopes suffer severe stray light issues. The telescope needs to be baffled to minimize stray light contamination at the focal plane within the field of view of the telescope. Without proper baffle design single reflection light paths either from the primary mirrors or the secondary mirrors generate stray light at the focal plane [3,4]. Typically, external set of baffles in front of the telescope have been used to minimize single reflection stray light $[4,5,6]$. Baffles inside the mirror cavities have also been studied [3,4], but these have not typically been implemented in the flight design.

Mangus [7] developed a baffling concept for Wolter Type 2 telescopes. He described the basic processes for determining the axial locations and radial heights of the baffles based on first order geometric principles. Baffle placement strategy is 
simply to limit the field of view, when viewed from the detector plane, to that of the secondary mirrors only. After the reflection on the secondary mirror, the view should be limited by baffles to the primary mirror only.

Wolter (W) type 1 telescopes [8] are typically used in x-ray astrophysics applications. These telescopes are stigmatic and offer an excellent, but narrow field of view. Type 1 hyperboloid-hyperboloid designs improve significantly the off-axis performance over larger field of views. Hyperboloid-hyperboloid designs [9] have been used for solar physics applications. Polynomial type 1 telescopes $[10,11,12,13]$ have been proposed for wide field of view astrophysics applications. These designs offer improved performance over larger fields of view.

Wolter-Schwarzschild (WS) type 1[14] telescopes are also stigmatic. This design has an excellent off-axis optical performance. WS designs satisfy Abbe's sine condition and are therefore free from coma aberration. Surface intersection point of the primary mirror and secondary mirror define the principal surface of the telescope. Principal surface of the WS telescopes is spherical in shape. Nested WS telescopes [15] should be placed on the principal surface. Then, all the nested telescopes have the same focal length and excellent off-axis performance. Absence of coma in WS telescopes improves the symmetry of off-axis Point Spread Functions (PSF). This is an important property when several telescopes are nested inside each other.

Surface equations of WS telescopes are complex parametric set of equations [16]. The equations can be expressed in terms of basic parameters such as focal length, physical length of the telescope, and maximum entrance aperture radius. Use of telescope basic dimensional parameters in the design work simplifies the setup and design of nested WS telescope systems [15].

\section{OPTICAL DESIGN}

Effective area, and field of view requirements of the STAR-X telescope drive available design options [1]. These requirements dictate the telescope focal length to be $5.0 \mathrm{~m}$, maximum outer diameter to be about $1.3 \mathrm{~m}$, effective area of the telescope at $1 \mathrm{KeV}$ to be above $2000 \mathrm{~cm}^{2}$, field of view to be 1 degree in diameter, and half power diameter (HPD) to be less than 5.0 arc-sec at $1.0 \mathrm{KeV}$. Stray light mitigation is also an important factor in the design. Full stray light control needs to be traded off against the effective area and resolution requirements.

\subsection{Optical Design}

STAR-X telescope design is based on a meta-shell concept [2]. In this approach, the mirror assembly is divided into many meta-shells. Each meta-shell is composed of many segments aligned and bonded into groups of shells. Basic building blocks of the meta-shells are segmented primary mirrors and secondary mirrors of Modified Wolter-Schwarzschild (MWS) telescopes [15]. Each segment is mounted on the backside of the adjacent inner segment using 4 mount posts in-between the segments.

Optical design space is limited by telescope focal length $(5.0 \mathrm{~m})$, primary and secondary mirror lengths $(100 \mathrm{~mm})$, and length of the tube-baffles in front of the telescope. Tube-baffles act also as thermal shields for the telescope assembly [2]. Primary and secondary mirror segments and meta-shells are separated by a small gap. The gap size of the STAR-X telescope is $6 \mathrm{~mm}$. The nested telescopes are designed so that the common principal surface of individual telescopes is located in the middle of the gap region. Meta-shells of the STAR-X telescope are assembled from 30-degree primary and secondary mirror segments.

Off-axis optical performances of individual MWS telescopes at best focal surfaces can significantly be improved over Wolter-Schwarzschild (WS) telescope designs [15]. The improvement is achieved by adding small amounts of axial second order sag either on the primary mirrors or on the secondary mirrors or both primary mirrors and secondary mirrors. Added axial sags spread the Point Spread Functions (PSF) uniformly across the field of view. The sags degrade the image near 
on-axis field points and improve the PSF closer to the outer field points. Off-axis improvements are achieved by the balancing act between aberrations caused by added axial sags and symmetric aberration components of WS telescopes. In the STAR-X nested design $0.21 \mu \mathrm{m}$ (PV) was added to all primary mirrors. The amount of added axial sag should to be the same for all the mirrors if the mirror pairs have the same focal length. The axial lengths of the mirrors need also to be the same across the stack of nested primary mirrors and secondary mirrors.

Average slope angle of the innermost primary mirror must be larger than the half-field of view ( 0.5 degrees). This requirement limits the radial height of the innermost telescope to about $174.6 \mathrm{~mm}$. Assuming mirror thickness of $0.4 \mathrm{~mm}$ and 1.0-degree field of view, 148 mirror pairs can be fit within the radial height limits.

\subsection{Baffle design}

Large field of view exposes the nested telescopes to severe stray light problems. Several sets of baffles are required to minimize stray light from reaching the detector of the telescope. We implemented baffle design principles described by Mangus [7]. Cross-section of the design is illustrated in Figure 1. Nested mirrors are designed so that when viewed from

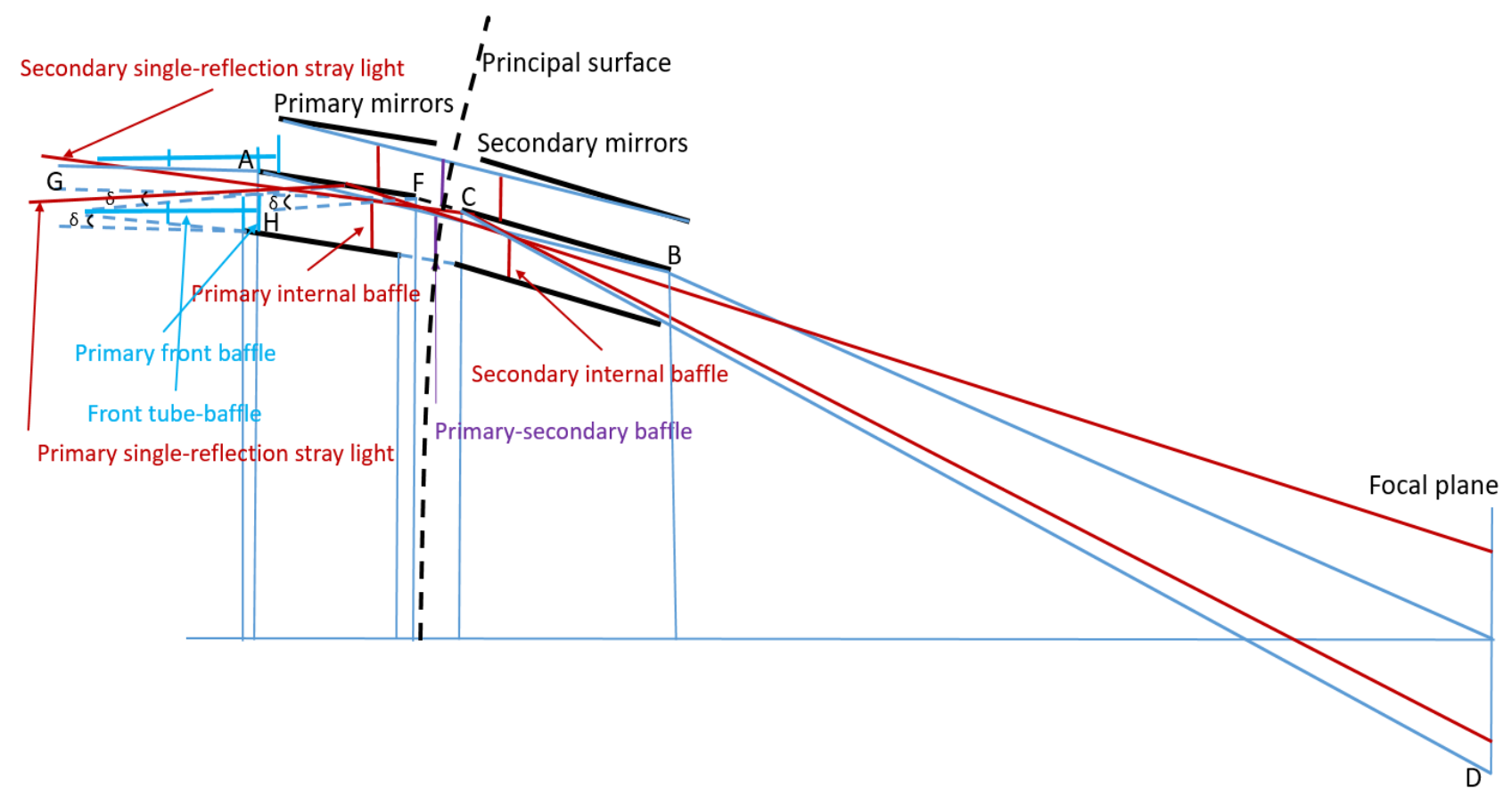

Figure 1. Cross-section of nested telescopes showing primary mirrors, secondary mirrors, and principal surface. Single-reflection stray light paths are also depicted as well as required baffles and limiting ray paths.

the detector plane only optical surfaces of secondary mirrors are seen. Radial heights of the cavities between the mirrors need to be set so that a ray D starting at the lower edge of the detector (-0.5-degree field point) and intersecting front corner of the secondary mirrors is not obstructed by the inner secondary mirrors. To minimize stray reflections, internal baffles of the secondary mirrors need to be placed in the cavities of secondary mirrors so that an on-axis rays A-B and extreme field rays C-D pass these baffles. Similarly, internal baffles of primary mirrors are placed at the intersections of on-axis ray A-B and G-F. Primary front baffles should obstruct the area below G-F ray and the primary mirror below. Primarysecondary baffles are placed at the primary-secondary surface intersection planes. The purpose of these baffles is to limit stray rays from entering adjacent secondary mirror cavities.

Front tube-baffles illustrated in Figure 1 are also needed to minimize the stray radiation within the field of view at the focal plane. There are single-reflection lines of sight that pass the internal baffles of secondary mirrors, and baffles of 
primary mirrors, and reach unobstructed the object space. Similarly, as illustrated in Figure 1, a line of sight starting within the detector field of view passes the internal baffles of the secondary mirrors, strikes the secondary mirrors and passes internal baffles of the primary mirrors.

Nesting density of the telescopes determines the axial length of the front tube-baffles. Thickness of the front tube-baffles in this design is $0.2 \mathrm{~mm}$. Dashed line H-F in Figure 1 illustrates a path from an extreme field point to the back of the primary mirror. If these paths would be unobstructed, the front tube-baffle would have to be very short. With short tubebaffles, it would be impossible to eliminate single-reflection stray light paths of the system. Instead, a path limited by onaxis ray G-F and primary front baffle are used to determine the lengths of the front tube-baffles. A small fraction of the off-axis effective area is lost due to this obscuration.

Following the principles presented above, our baffle design tool calculates the radial heights and axial locations of the primary and secondary baffles. Maximum lengths of the front tube-baffles are also calculated. The tool traces optical paths in the meridional plane starting from the focal plane backwards towards the secondary mirrors and checks if the paths are obstructed by back corners of the secondary mirrors and secondary internal baffles. After the reflection on the secondary mirror, the tool checks if the paths are obstructed by the baffles of primary mirrors and front tube-baffles. Similarly, the tool checks if the paths from the detector plane within the field of view to the primary mirrors are obstructed by secondary mirror baffles. After reflection on the primary mirrors, the tool checks if the paths are obstructed by the baffles of the primary mirrors and the lower front tube-baffle.
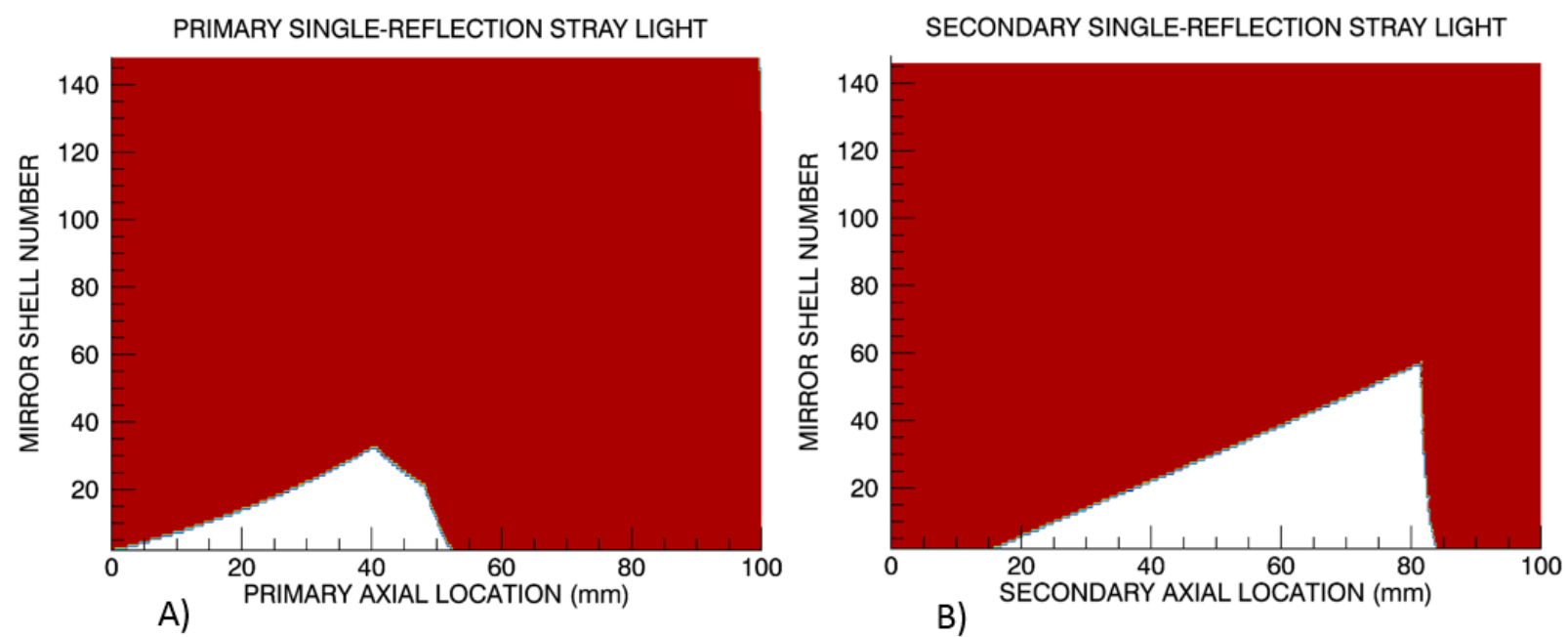

Figure 2. White axial areas represent exposed mirror surface area for single-reflection stray light of A) the primary mirrors and B) secondary mirrors.

STAR-X design described in Section 2.1 cannot be completely baffled for single-reflection stray light. Axial lengths of the front tube-baffles are approximately $63 \mathrm{~mm}$ for every mirror pair. Figure 2.A shows the exposed axial lengths of the primary mirrors. Front half of the innermost shells are exposed to stray light. Unobstructed areas decrease as a function of the shell number. As you can see in Figure 2.A, the thirtieth mirror pair is not exposed to stray light. At the focal plane, single-reflection rays would contaminate a toroidal area from 0.28 degrees to 0.5 degrees. Central 0.28 degrees of the detector plane would not be contaminated by single-reflection stray light of the primary mirrors.

Figure 2.B shows the exposed axial areas on the secondary mirrors as a function of mirror shell number. Exposure on the secondary mirrors is much worse. Exposed axial areas extend up to $80 \mathrm{~mm}$ of $100 \mathrm{~mm}$ long secondary mirrors. Number of the exposed innermost shells is 77. At the focal plane, single-reflection rays would contaminate a toroidal area from 0.16 degrees to 0.5 degrees. Central 0.16 degrees would not be contaminated. 
To eliminate single-reflection stray light paths, the internal primary and secondary mirror baffles of the effected mirror pairs would have to be made taller. Unfortunately, this process would decrease the effective area of the system. Alternative way is to increase the spacing between the mirror pairs in the effected range. Increased spacing between the mirror pairs make it possible to increase the axial length of the front tube-baffle and eliminate single-reflection stray light paths. Relaxed packing density of the mirrors will also decrease the effective area.

\section{DIMENSIONS OF THE BAFFLES AND PERFORMANCE OF THE TELESCOPE}

In the STAR-X design we selected 101 outermost mirror pairs and increased the spacing of 29 innermost mirror pairs to eliminate the single-reflection stray light. Larger cavities between the innermost shells will lower the effective area especially at higher energies. This design still meets the effective area requirements of the STAR-X telescope.

\subsection{Dimensions of the baffle system}

Figure 3.A plots the axial lengths of the front tube-baffles as a function of mirror shell number. Increased spacing of 29 innermost mirror pairs significantly increase the length of the tube. Innermost tube is about $114 \mathrm{~mm}$ long. Axial length of
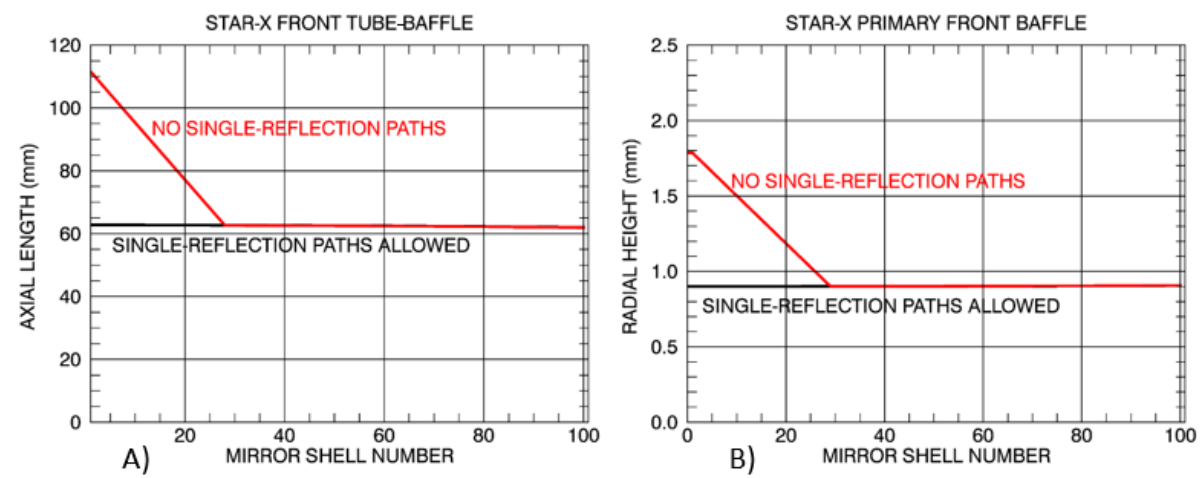

Figure 3. A) Axial lengths of the front tube-baffle of the nested mirror pairs. B) Radial heights of the front baffles of the primary mirrors. Axial lengths and radial heights are shown for the designs that allows and eliminates single-reflection stray light.

the outermost mirror pairs that are not spread out are about $63 \mathrm{~mm}$ long.

Figure 3.B shows the radial heights of the front baffles of the primary mirrors. The increase of the spacing of inner 29 mirror pairs increase the radial height of the baffles. The height of innermost pair doubles to $1.8 \mathrm{~mm}$. Front baffles of the

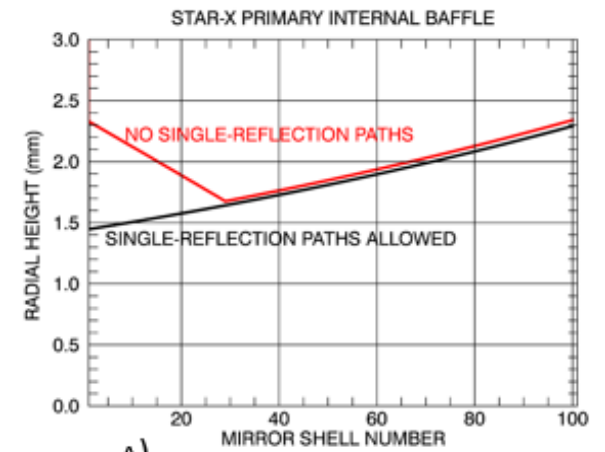

A)

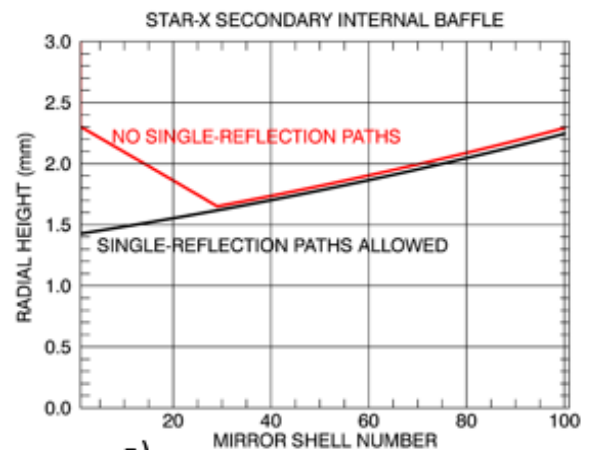

B)

Figure 4. Radial heights of the internal baffles of A) nested primary mirrors and B) nested secondary mirrors. Radial heights are shown for the designs that allow and eliminate single-reflection stray light. 
primary mirrors are positioned in the front edge of the primary mirrors. These baffles could be integrated to the front tubebaffles.

Radial heights of the internal baffles of primary mirrors and internal baffles of the secondary mirrors are shown in Figure 4.A and 4.B. Figure 5.A plots the axial position of these internal baffles. Again, the increased cavity size between the 29

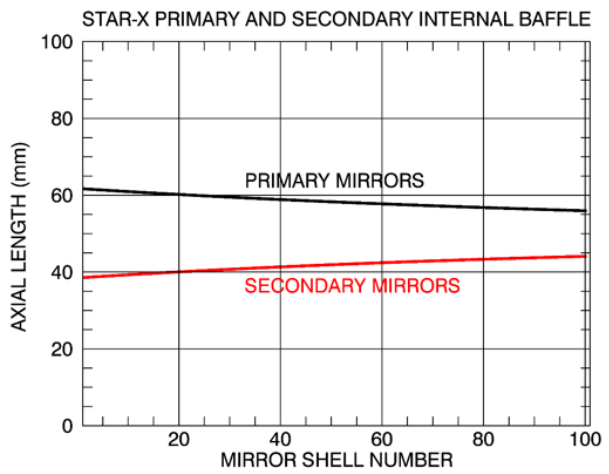

Figure 5. Axial locations of primary and secondary internal baffles.

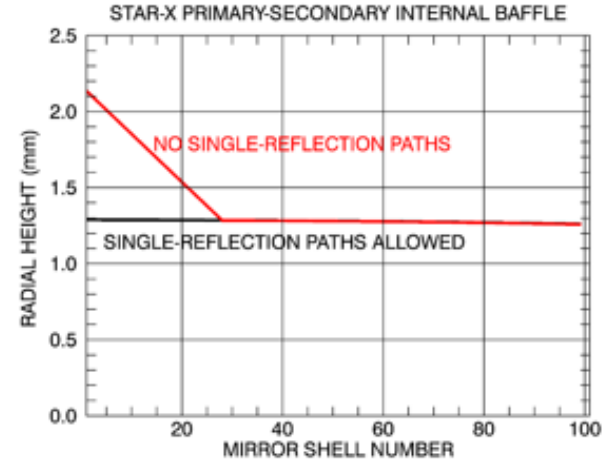

Figure 6. Radial heights of the baffles at the intersections of the primary and secondary mirrors.

innermost mirror pairs increase the radial heights. Axial locations of the internal baffles change a few millimeters throughout of the nested mirrors. Internal baffles of the primary mirrors move towards the front of the mirror and internal baffles of the secondary mirrors move towards the backend of the secondary mirrors. Internal baffles need to be mounted on the backside of the mirrors increasing a risk of deforming the mirror in the mounting process. Baffles would have to be made from the mirror material to minimize thermal effects.

Figure 6 plots the radial heights of primary-secondary baffles at the plane of intersection of the surfaces as a function of the mirror shell number. Radial heights remain nearly unchanged in case of outer mirror pairs but increase sharply in case of inner mirror pairs where the spacing between the mirrors is increased to eliminate the single-reflection stray light.

\subsection{Optical performance of the telescope}

Optical performance of the STAR-X telescope was evaluated at $1.0 \mathrm{KeV}$ and $4.0 \mathrm{KeV}$ energies. Iridium coatings on the optical surfaces were assumed. Optical constants published by Lawrence Berkeley Laboratory [17] were used to calculate reflection losses at the mirror surfaces. Both WS telescope and MWS telescope consisting of 101 mirror pairs were evaluated by ray tracing and, then, calculating Half Power Diameter (HPD) and the effective area of the telescopes at the
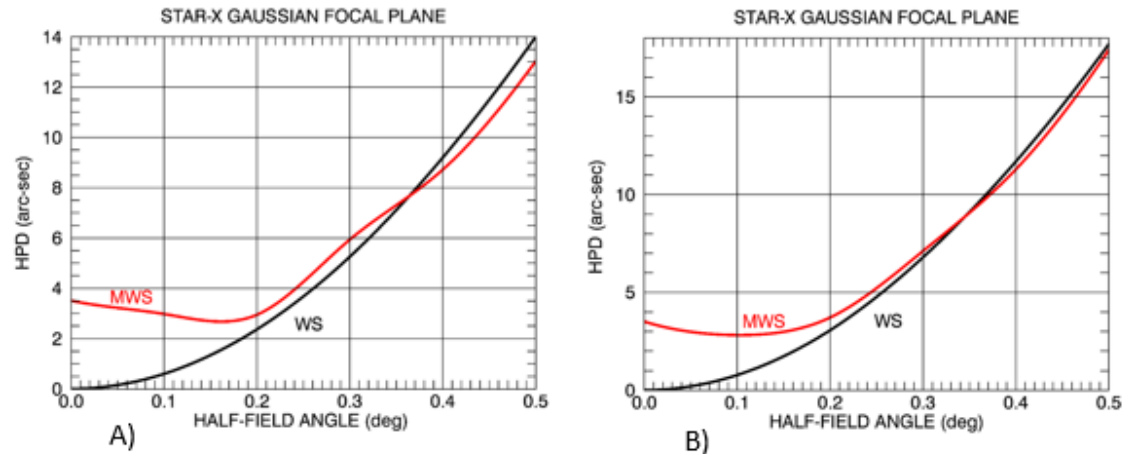

Figure 7. HPD of the WS and MWS telescope calculated at the Gaussian focal plane at A) $1.0 \mathrm{KeV}$ and B) 4.0 $\mathrm{KeV}$. 
selected energies. No image degradation from alignment errors of the mirrors or surface figure errors of the mirrors were assumed. Segmented nature of the meta-shells or obscurations caused by the mount posts of the mirror segments were not included in the ray trace results.

Figures 7.A and 7.B plot the HPD as a function of the half-field angle at the Gaussian focal plane for $1.0 \mathrm{KeV}$ and 4.0 $\mathrm{KeV}$ energies, respectively. HPDs of the telescopes increase to about 15 arc-sec over the 0.5 -degree field. The WS design provides better performance within 0.35 degrees. The MWS telescope has just slightly better performance in the range from 0.35 degrees to 0.5 degrees.
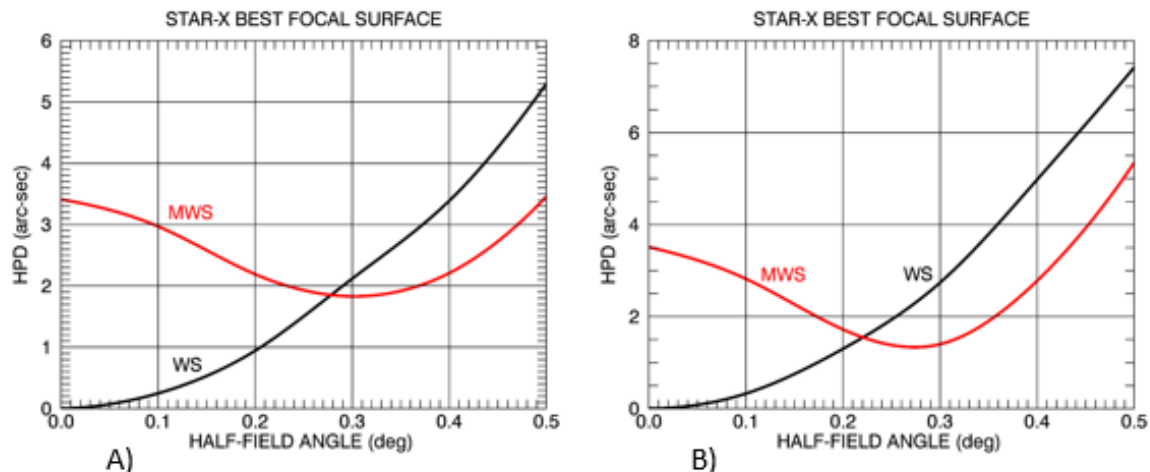

Figure 8. HPD of the WS and MWS telescope calculated at the best focal surface at A) $1.0 \mathrm{KeV}$ and B) $4.0 \mathrm{KeV}$.

Optical performance of the telescope at the best focal surface is shown in Figures 8.A and 8.B for energies $1.0 \mathrm{KeV}$ and $4.0 \mathrm{KeV}$, respectively. HPD of WS design increases across the field of view to about $5.3 \mathrm{arc}-\mathrm{sec}$ and 7.4 arc-sec at 1.0 $\mathrm{KeV}$ and $4.0 \mathrm{KeV}$, respectively. HPD of the MWS telescope is more uniform across the field of view. Added axial sags increase the on-axis HPD to 3.4 arc-sec and lower the HPD at the edge of the field to 3.4 arc-sec at $1.0 \mathrm{KeV}$ energy. HPD reaches minimum of 1.8 arc-sec at 0.31 degrees off-axis. At $4.0 \mathrm{KeV}$, the $\mathrm{HPD}$ is not as uniform as at $1.0 \mathrm{KeV}$. HPD ranges from 3.5 arc-sec to 5.3 arc-sec at the edge of the field. HPD-curve has a minimum of $1.3 \mathrm{arc}-\mathrm{sec}$ at 0.28 -degree offaxis location. A slight adjustment in the axial sags of mirrors would result in a more uniform HPD across the field at 4.0
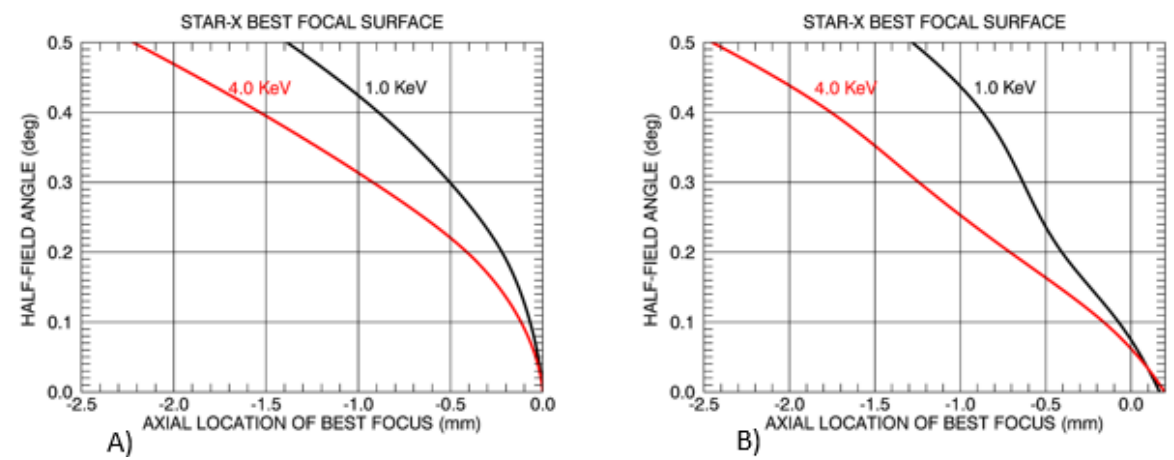

Figure 9. Half-field angle as a function of best focus at $1.0 \mathrm{KeV}$ and $4.0 \mathrm{KeV}$ for A) WS telescope and B) MWS telescope.

$\mathrm{KeV}$.

In Figures 9.A and 9.B, the half-field angle is plotted as a function of the axial location of the best focal surface for WS and MWS telescopes, respectively. At higher energies, inner mirror pairs contribute more because the reflectance of outer mirror pairs is reduced. On the other hand, the field curvature of inner mirror pairs is much worse than the field curvature of outer mirror pairs. Added axial sags of the MWS telescope tend to flatten the shape of the best focal surface. Aberrations caused by added axial sags are dominating image defect in the field points near optical axis. 
Effective area of the STAR-X telescope is shown in Figure 10 at 1.0 $\mathrm{KeV}$ and $4.0 \mathrm{KeV}$. No structural obscurations are assumed when calculating the effective area. These obscurations typically decrease the effective area $25 \%$ to $30 \%$ in a nested x-ray telescopes. At $1.0 \mathrm{KeV}$, on-axis effective area is above $4000 \mathrm{~cm}^{2}$. It drops nearly linearly to $2400 \mathrm{~cm}^{2}$ towards the edge of the field of view. Vignetting is the driving factor that decreases the off-axis effective area. In this design, secondary mirrors are designed to pass just the on-axis rays. Part of the off-axis rays hitting front and back circumference of the primary mirrors are vignetted. Front baffles of the primary mirrors partially obstruct the field of view as illustrated in Figure 1 by rays H-F.

Large field curvature of the grazing incidence telescopes limits the field of view of the telescope. If 1.0-degree field of view is required, multiple detectors are need to improve the optical performance. With multiple detectors, the best focal surface can be matched well. Reverse pyramid configuration with 4 detectors is one of the simplest options [18]. In Figure 11 , the HPD is shown for a reverse pyramid configuration. The detectors are positioned so that the tip of the pyramid is placed on-axis at the telescope focus, while the 0.5-degree off-axis (best focus) location is also on the detector diagonal axis. The HPDs calculated at $1.0 \mathrm{KeV}$ are plotted for nested MWS-telescope along the diagonal axis and along edge of the detector. The HPD of the nested MWS-telescope is below 5 arc-sec along the diagonal direction and slightly over 5-arc-sec at the edge of the field along the edge of the detector. An array of 8 detectors would be needed to keep the HPD below 5-arc-sec.

\section{CONCLUSIONS}

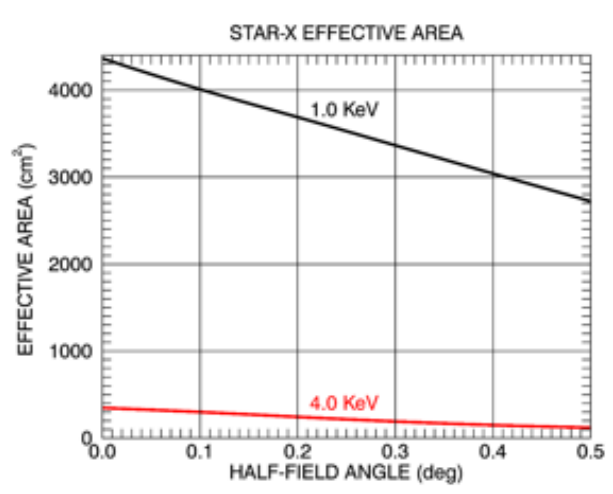

Figure 10. Effective area of the STAR-X telescope at $1.0 \mathrm{KeV}$ and $4.0 \mathrm{KeV}$.

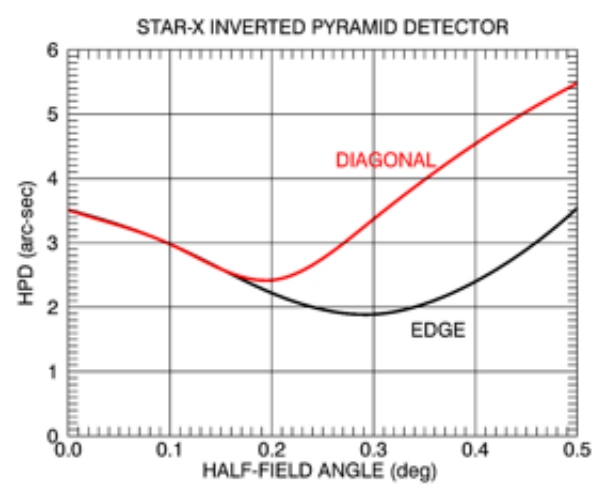

Figure 11. HPDs of STAR-X telescope at 1.0 $\mathrm{KeV}$ assuming the 4 detectors are placed in inverted pyramid configuration.

Tightly nested and wide field of view grazing incidence telescopes cannot be completely baffled. Especially, inner mirror pairs would require very long tube-baffles in front of the telescope. Long tube-baffles would increase significantly the total length of the instrument. Increased length of the tube-baffles would also force the designer to increase the radial separation of the nested mirror pairs and, thus, reduce the effective area of the telescope. For this reason, the telescope baffling design should always be integral part of the telescope optical design.

Type $1 \mathrm{x}$-ray telescopes are prone to single-reflection stray light problems. External tube-baffles and internal primary and secondary mirrors baffles are needed to prevent single-reflection stray rays reaching the focal plane within the field of view of the telescope. Baffling assembly of the telescope can be very complex since every nested pair of mirrors require its own baffles.

Structural components separating the nested mirrors in the meta-shell and small gaps between the mirror segments need also to be baffled. These baffles can be incorporated to baffles of the telescope placed in front of the primary mirrors and secondary mirrors.

Nested grazing incidence telescope are structurally very complex and challenging to baffle. Baffle system presented here were designed to eliminate mainly direct and single-reflection stay light contamination. There 
can also be multiple-reflection stray light paths from optical surfaces, from backside of the mirrors, or combination of baffles, mirrors, and structural components. A thorough stray light analysis using sophisticated stray light analysis tools should be completed to understand level of contamination caused by stray light problems of the nested telescope.

\section{ACKNOWLEDGEMENTS}

This work has been financially supported by the Next Generation X-Ray Optics Project at Goddard Space Flight Center in Greenbelt, Maryland.

\section{REFERENCES}

[1] William W. Zhang, "The survey and time-domain astrophysical research explorer (STAR-X), "Proc. of SPIE 10397-25 (2017)

[2] Ryan S. McClelland, Timo T. Saha, William W. Zhang, Peter M. Solly, and Joseph Bonafede, "The STAR-X x-ray telescope assembly, "Proc. of SPIE 10399-7 (2017)

[3] Edward C. Moran and James E. Harvey, "Ghost image behavior in Wolter type x-ray 1 telescopes," Appl. Opt. 27, 1486-1491 (1988).

[4] M.V. Zombeck, G.K. Austin, and D.T.Torgenson, "Advanced x-ray astrophysics facility (AFAF) interim engineering report, SAOAXAF-80-003 (1980).

[5] Daniel de Chambure, Robert Laine, Kees van Katwijk, Wolfgang Ruhe, Dietmar Schnik, Edgar Hoelzle, Yoland Gutierrez, Miguel Domingo, Inigo Ibarretxe, Jean Philippe Tock, Isabelle Domken, Yvan Stockman, Yvette Houbrecht, Herbert Hansen, and Bernd Aschenbach, "The x-ray baffle of the XMM telescope: development and results, "Proc. of SPIE, 3737, 396-408 (1999).

[6] Eric Wille, Marcos Bavdaz, Sebastian Fransen, Mazimilien Collon, Maecelo Ackermann, Ramses Guenther, Giuseppe Vacanti, Coen Van Baren, Jeroen Haneveld, Mark Olde Riekerink, Arenda Koelewijn, Dirk Kampf, Karl-Heinz Zuknik, and Arnd Reutlinger, "Stray light bafflinf and Environmental Qualification of silicon pore optics, "Proc. of SPIE 8861, 88611E-1- 8861E-7.

[7] John D. Mangus, "Strategy and calculations for the design of baffles for Wolter type ll telescopes, "Proc of SPIE 830, 245-253 (1987).

[8] L. P. Van Speybroeck and R.C. Chase, "design parameters of paraboloid-hyperboloid telescopes for x-ray astronomy, Appl. Opt. 11, 440-445 (1972).

[9] James E. Harvey, Andrey Krywonos, Patrick L. Thompson, and Timo T. Saha, "Grazing incidence hyperboloid-hyperpoloid designs for wide-field x-ray imaging applications,"Appl. Opt., 40, 136-144 (2001).

[10] Christopher J. Burrows, Richard Burg, and Riccardo Giacconi, “Ap.J., 392, 760-765 (1992).

[11] Paolo Conconi, Sergio Campana, Gianpiero Tagliaferri, Giovanni Pareschi, Obertto Citterio, Vincenzo Cotroneo, Laure Proserpio, and Martha Civitani, "A wide field X-ray telescope for astronomical survey purposes: from theory to practice, "Mon. Not. R. Astron. Soc., 405, 877-886 (2010).

[12] Ronald F. Elsner, Stephen L. O’Dell, Brian D. Ramsey, and Martin C. Weisskopf, "Methods of optimizing x-ray optical prescriptions for wide-field applications, "Proc. of SPIE, 7732, 77322L-77322L-14 (2010).

[13] Peter. W .A. Roming, John C. Liechty, David H. Sohn, Jared R. Shoemaker, David N. Burrows, Gordon P. Garmire, "Markov chain Monte Carlo algorithms for optimizing grazing incidence optics for wide-field x-ray survey imaging, "Proc. of SPIE, 4496, 146-153 (2002). 
[14] R. C. Chase and L. P. VanSpeybroeck, "Wolter-Schwarzschild telescopes for x-ray astronomy, “Appl. Opt., 12, 1042 1044 (1973).

[15] Timo T. Saha, Ryan S. McClelland, and William W. Zhang, "Optical Design for a survey telescope, "Proc. of SPIE 9144, 914418- 914418-914418-12 (2014).

[16] Timo T. Saha, “General surface equations for glancing incidence telescopes, “Appl. Opt., 26, 658-663 (1987).

[17] http://henke.lbl.gov/optical_constants/

[18] Ronald F. Elsner, Stephen L. O'Dell, Brian D. Ramsey, and Martin C. Weisskopf, "Mathematical formalism for designing wide-field x-ray telescopes: mirror nodal positions and detector tilts, "Proc. of SPIE, 8147, 814712-1-81471215 (2011). 